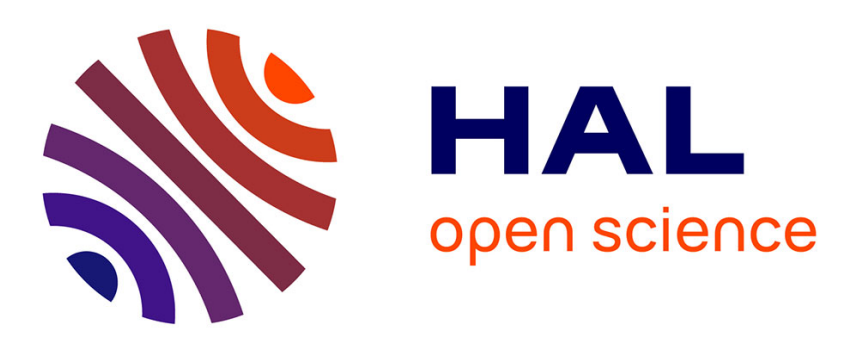

\title{
Motor endplate disease affects neuromuscular junction maturation
}

Ghislaine Caillol, Hélène Vacher, Magali Musarella, Sarah Bellouze, Bénédicte Dargent, Amapola Autillo-Touati

\section{To cite this version:}

Ghislaine Caillol, Hélène Vacher, Magali Musarella, Sarah Bellouze, Bénédicte Dargent, et al.. Motor endplate disease affects neuromuscular junction maturation. European Journal of Neuroscience, 2012, 36 (4), pp.2400 - 2408. 10.1111/j.1460-9568.2012.08164.x . hal-01701550

\section{HAL Id: hal-01701550 \\ https://hal.science/hal-01701550}

Submitted on 20 Apr 2018

HAL is a multi-disciplinary open access archive for the deposit and dissemination of scientific research documents, whether they are published or not. The documents may come from teaching and research institutions in France or abroad, or from public or private research centers.
L'archive ouverte pluridisciplinaire HAL, est destinée au dépôt et à la diffusion de documents scientifiques de niveau recherche, publiés ou non, émanant des établissements d'enseignement et de recherche français ou étrangers, des laboratoires publics ou privés. 


\title{
Motor endplate disease affects neuromuscular junction maturation
}

\author{
Ghislaine Caillol, ${ }^{1,2, \star}$ Hélène Vacher, ${ }^{1,2}$ Magali Musarella, ${ }^{1,2}$ Sarah Bellouze, ${ }^{1,2}$ Bénédicte Dargent ${ }^{1,2}$ \\ and Amapola Autillo-Touati ${ }^{1,2}$ \\ ${ }^{1}$ Institut National de la Santé et de la Recherche Médicale, Unité Mixte de Recherche 641, Marseille 13916, France \\ Université de la Méditerranée, Institut Fédératif de Recherche 11, Marseille 13916, France
}

Keywords: med mouse, synaptogenesis, terminal Schwann cells

\begin{abstract}
Postnatal formation of the neuromuscular synapse requires complex interactions among nerve terminal, muscle fibres and terminal Schwann cells. In motor endplate disease (med) mice, neuromuscular transmission is severely impaired without alteration of axonal conduction and a lethal paralytic phenotype occurs during the postnatal period. The med phenotype appears at a crucial stage of the neuromuscular junction development, corresponding to the increase in terminal Schwann cell number, the elimination of the multiple innervations and the pre- and postsynaptic maturation. Here we investigated the early cellular and molecular consequences of the med mutation on neuromuscular junction development. We observed that cellular defects preceded overt clinical phenotype. The first detectable cellular effect of the mutation at the onset of the clinical phenotype was a drastic reduction in the number of terminal Schwann cells, in part due to an increase in glial apoptosis, and a delayed maturation of motor endplates. We also showed that, in terminally ill animals, mono-innervation was not achieved, synaptic vesicles had accumulated in the presynaptic compartment and, finally, the size of motor endplates was reduced. All together, our findings suggested that the clinical weakness in these mutant mice was likely to be related to postnatal structural abnormalities of the neuromuscular junction maturation.
\end{abstract}

\section{Introduction}

Motor endplate disease (med) is a severe genetic disorder in mice (Duchen et al., 1967; Duchen, 1970). Homozygous med mutants suffer from progressive muscular weakness and cerebellar ataxia, leading to paralysis and premature death within the first month of life. Electrophysiological studies show that paralysis is caused by a secondary 'functional denervation' of skeletal muscle (Duchen \& Stefani, 1971; Angaut-Petit et al., 1982; Kearney et al., 2002), as mutant mice do not express a reduction in motor neurons in the spinal cord (Porter et al., 1996). There is actually excess sprouting of motor nerve terminals (Brown et al., 1980; Musarella et al., 2006) similar to that associated with failed neuromuscular transmission due to intracellular injections of botulinum toxin (Duchen \& Strich, 1968). Furthermore, neuromuscular transmission is impaired without alteration of axonal conduction, at least down to the intramuscular branches (Duchen \& Stefani, 1971). Med mutant ataxia is also associated with a loss of spontaneous electrical activity in Purkinje cells (Dick et al., 1985).

Correspondence: Amapola Autillo-Touati, ${ }^{1}$ Institut National de la Santé et de la Recherche Médicale as above.

E-mail: amapola.autillo-touati@univ-amu.fr

*Present address: UMR 7286 CNRS-Aix-Marseille Université, Faculté de Médecine Nord, boulevard Pierre Dramard, 13916 Marseille Cedex 20, France.

Received 11 July 2011, revised 11 April 2012, accepted15 April 2012
Transgene-induced mutation at the mouse med allele induces a loss of expression of the voltage-gated sodium channel gene Scn8a encoding for Nav1.6 (Burgess et al., 1995; Kohrman et al., 1996). Nav1.6 is expressed in mature peripheral and central nerves (Caldwell et al., 2000; Boiko et al., 2001) as well as in terminal Schwann cells (TSCs), also known as perisynaptic Schwann cells, at neuromuscular junctions (NMJs; Musarella et al., 2006). NMJs, commonly called tripartite synapses (Araque et al., 1999), are defined by physical and functional interactions of glia with preand postsynaptic elements, where TSCs are an integral modulatory partner (for reviews Todd \& Robitaille, 2006; Feng \& Ko, 2008a). TSCs actively participate in the formation, maintenance and repair of the neuromuscular synapse, and modulate neuromuscular transmission (Son et al., 1996; Rochon et al., 2001; Auld \& Robitaille, 2003; Kang et al., 2003; Reddy et al., 2003; Feng et al., 2005; Feng \& Ko, 2008b; Todd et al., 2010). Strikingly, in terminally ill med mice [postnatal day (P) 19], the number of TSCs per NMJ is dramatically decreased (Musarella et al., 2006) and, in contrast to neurons (Van Wart \& Matthews, 2006; Vega et al., 2008), no compensatory expression of other sodium channels occurs in these TSCs (Musarella et al., 2006).

Based on these observations, we raised questions as to whether the med mutation could induce structural abnormalities of the NMJ leading to pathology of the NMJ development. In this study we investigated, using confocal microscopy, 3-D reconstruction and 
quantification, how the postnatal development of the three cellular partners of the NMJ is affected in the med phenotype. We showed that med mice NMJ development is affected by progressive loss of TSCs and by halting maturation of the synaptic structure.

\section{Materials and methods \\ Animals}

All work was performed under the responsibility of an authorized experimenter (A.A.-T., license no. 13-09), in accordance with the European Communities Council Directive (86/809/EEC) on the care and use of animals for experimental procedures, and was approved by local ethical committee of IFR (Institut Fédératif de Recherche) 11, Faculty of Medicine, Marseille. Jackson Laboratories (USA) supplied initial breeder pairs of mice. The med is a hereditary disorder transmitted by an autosomal recessive gene (med). Med allele was maintained by crossing heterozygous mice. All the animals were genotyped by PCR from tail and littermate wild type (WT) were used as controls.

Physical and functional features of disease onset and progression in med mice were characterized by careful examination of $>100$ litters. According to Duchen (1970), four features were defined: failure of thrive, muscular atrophy, nodding movement of the head and finally crawling. From birth to 8 or 9 days, homozygous med mice could not be distinguished from their normal or heterozygous littermates, and P7 was chosen to illustrate this period. The first signs of abnormality appeared at P10 (see Movie S1, supporting information) with a failure to thrive and a beginning of muscular atrophy affecting especially the posterior limbs. Consequently, med mice moved more slowly than WT and heterozygous mice. The mutant mice swayed slightly from side to side and crawled with a light movement of the head. Within the next few days, the proximal muscles of all limbs were also affected. At P19-20, the muscular weakness and atrophy were so severe that the med mice could not make any effective movements (see Movie S2, supporting information), leading to a premature death. Therefore, we selected three ages according to the development of the med paralytic phenotype: P7 (before the onset of the phenotype), P10 (onset of the phenotype) and P19 (terminally ill phenotype).

\section{Muscle preparation}

All the mice from P7 to P19 were deeply anesthetized with pentobarbital (120 mg/kg b.w., i.p.) before dissecting them. Sternal triangularis (ST) and sternomastoid (SM) muscles from P7, 10 and 19 med and WT mice were dissected in an oxygenated Ringer solution (95\% O2, 5\% CO2) and fixed for $20 \mathrm{~min}$ in $4 \%$ paraformaldehyde in phosphate buffer (PB), $\mathrm{pH}$ 7.4. Cryopreservation was carried out overnight at $+4{ }^{\circ} \mathrm{C}$ prior to freezing on liquid natrium vapour. SM muscles were mounted in Tissue-Tek*Cryo-O.C.T (Optimum Cutting Temperature) Compound (Miles, USA) and serial cryostat sections of $40 \mu \mathrm{m}$ were cut. Whole-mount of ST and SM muscle sections were rinsed in $0.1 \mathrm{M} \mathrm{PB}, \mathrm{pH} 7.4$, then in $10 \mu \mathrm{g} / \mathrm{mL}$ bovine serum albumin (BSA) in PB and incubated for $1 \mathrm{~h}$ in $50 \mu \mathrm{g} / \mathrm{ml} \mathrm{BSA}$ with $0.5 \%$ Triton X-100 in PB. They were then incubated for $2 \mathrm{~h}$ at room temperature (RT) with primary antibodies in $10 \mu \mathrm{g} / \mathrm{mL}$ BSA in PB, and rinsed. Fluorochrome-conjugated secondary antibodies and fluorochrome-conjugated ligands were then added for $1 \mathrm{~h}$. In some muscle preparations, DAPI nucleic acid stain $(1: 24000,0.1 \mu \mathrm{M}$; Molecular Probes, Eugene, OR, USA) was added for $10 \mathrm{~min}$ at RT prior mounting. Preparations were mounted with Fluorsave mounting medium (Molecular Probes).

\section{Antibodies and conjugated ligands}

Neurofilament $\mathrm{H}$ (NF) was detected using a rabbit polyclonal antiserum (1 : 100; Sigma Aldrich, Saint Louis, MO, USA) and an Alexa 488-conjugated goat antirabbit secondary antibody (1:600; Molecular Probes). Synaptic vesicle protein 2 (SV2) was immunostained using a mouse monoclonal antibody developed by Buckley \& Kelly (1985) (1 : 200; Developmental Studies Hybridoma Bank, Iowa City, IA, USA) and a Cy5-labelled donkey antimouse secondary antibody (1 : 400; Jackson Laboratories, West Grove, PA, USA). Schwann cells were detected by staining S100, a specific glial antigen (polyclonal anti-S100b primary antibody, $1: 150$; Dako) and a Cy5conjugated donkey antirabbit antibody (1:600; Jackson Laboratories). Nicotinic acetylcholine receptors (nAChRs) were visualized using a tetramethylrhodamine-conjugated $\alpha$-bungarotoxin $(\alpha$-BgTx, 1 : 200; Molecular Probes). Acetylcholine esterase was detected using an Alexa 488-conjugated fasciculin2. Conjugation and purification of fasciculin2 (Latoxan, France) with the Alexa dye was performed using the Molecular Probes Alexa Fluor ${ }^{\circledR} 488$ protein labeling kit and following the manufacturer's instructions. The conjugated fasciculin2 Alexa dye was purified from unconjugated dye by size exclusion chromatography using Bio-Gel P-4 (Bio-Rad). The dye attached to one or more lysine residues in the protein at the ratio of one mol of Alexa 488 attached per mol of fasciculin2. nAChR $\gamma / \delta$ subunits were detected using a monoclonal antibody from ascite (Antibodiesonline.com). Tubulin was detected using a mouse monoclonal antibody clone E7 anti- $\beta$-tubulin from the Developmental Studies Hybridoma Bank.

\section{Combined in situ cell death detection (TUNEL method) and immunostaining}

SM muscles cryostat sections from P7, P10 and P19 homozygous med and WT mice were rinsed in PB and incubated at $37{ }^{\circ} \mathrm{C}$ for $1 \mathrm{~h} 30 \mathrm{~min}$ in $50 \mu \mathrm{g} / \mathrm{mL}$ BSA with $0.3 \%$ Triton X-100 in PB. After rinsing, in situ detection of apoptotic cells was performed by using the In Situ Cell Death Detection Kit Fluorescein (Roche) and following the manufacturer's instructions. After rinsing, sections were incubated in a humidified chamber for $1 \mathrm{~h} 30 \mathrm{~min}$ in the dark, at RT, with an antiS100b rabbit polyclonal antibody and then with Cy5-conjugated donkey anti-rabbit antibody and conjugated $\alpha$-BgTx as described above. The sections were rinsed in PB and incubated with DAPI for $10 \mathrm{~min}$ at RT. Preparations were mounted as described above.

\section{Confocal acquisition, deconvolution imaging, 3-D rendering and quantification}

Confocal image acquisition was performed on a Leica DML 2500 and on a Leica TCS SP2 laser scanning microscope (Leica Microsystems, Heidelberg, Germany) with a $63 \times$ oil-immersion objective (numerical apertures 1.30 and 1.32). Pinhole size was set to 'Airy one' to achieve the best possible resolution (without reducing luminosity). On the Leica TCS SP2 laser scanning microscope, voxel size was adjusted to $58 \mathrm{~nm}$ in $x$ and $y$ and to $162 \mathrm{~nm}$ in $z$ by adjusting the scanning zoom factor. For each channel, photomultiplier gains and offsets were adjusted in order to use the full image dynamic range. Maximum intensity projections were used to represent confocal stacks. The depth of each confocal acquisition was adapted to explore the entire NMJ and incoming nerve fibre. Maximum intensity projections were used to represent confocal stacks. Deconvolution of confocal stacks and 3-D visualization was performed on a Silicon Graphics Octane 2 workstation. Point spread function (PSF) of the microscope was obtained 
from TetraSpeck fluorescent microsphere standards (Molecular Probes). To obtain high quality restoration of images, Huygens professional software was used (Scientific Volume Imaging, Hilversum, The Netherlands) with measured PSF and MLE (maximum likelihood estimation) algorithms. Three-dimensional rendering and measurement of active zone (SV2) volume were performed on Imaris software (Bitplane AG, Saint Paul, MN, USA). Area measurement was done with the integrated morphometric analysis tool from Universal Imaging Metamorph. The outer border of the $\alpha$-BgTx staining determined the motor endplate area.

\section{Immunoblotting analysis}

Total proteins were extracted from P19 frozen paraformaldehyde-fixed SM muscles from WT and med mice using the Qproteome FFPE tissue kit following the manufacturer's instructions (Qiagen). The concentration of the extracted proteins was determined by the BCA method (Pierce). An equal volume of reducing SDS sample buffer $(2 \times)$ was added to these extracted proteins. Sixty micrograms of total proteins of each sample were boiled and fractioned on SDS-9\% polyacrylamide gels. Gel electrophoresis and immunoblotting have been described (Vacher et al., 2007). After incubation with primary antibodies, blots were incubated with horseradish peroxidase-conjugated secondary antibodies (GE Healthcare) followed by enhanced chemiluminescence reagent (Perkin Elmer, Wellesley, MA, USA). Immunoreactive bands were visualized by exposing blots to X-ray film. Two independent sets of experiments were performed.

\section{Statistical analysis}

Means of motor endplate area, of volume occupied by SV2 and of TSC number per NMJ were statistically analyzed with unpaired Student's $t$-tests. Errors are given as mean \pm SD as noted. Statistically significant differences in the number of NMJs innervated according the parameters studied and with TSCs were calculated using Fisher's exact test. With large samples, a Chi-squared test was used (apoptotic nuclei number). Differences were considered significant at $P<0.05$. Data were statistically analyzed with GRAPH PAD PRISM Software.

\section{Results}

\section{Motor axonal pattern of the NMJs was altered in med mice at P19}

Med mice do not express a reduction in motor neurons in the spinal cord (Porter et al., 1996). To determine whether axonal defects might explain the weakness in these mutants we examined NMJs from whole-mount ST muscles from P19 WT and med mice (terminally ill animals as defined in the Material and methods section) with confocal microscopy. Antibodies against NF and SV2 proteins were used to visualize distal axons and nerve terminals, respectively, and nAChRs were stained with conjugated $\alpha$-BgTx. First, we quantified the number of axons innervating individual motor endplates that could be observed in their totality on two and three ST muscles from P19 WT and med animals, respectively (Table 1; Fig. 1G-I). Out of 196 junctions examined in the WT, $96 \%$ of the NMJs were monoinnervated and only $4 \%$ displayed persistent multiple innervation. In the mutant, $14 \%$ of the 326 junctions examined presented persistent multiple innervation (Table 1). The difference between multi- to mono- innervation in med and WT mice was significant $(P<0.05$, Fischer test), suggesting that the process of synapse elimination
TABLE 1. Analysis of NMJ innervation on ST muscles from P19 WT and med mice

\begin{tabular}{lcc}
\hline & \multicolumn{2}{l}{$\begin{array}{l}\text { NMJs: (\%) identified by NF/ } \\
\alpha \text {-BgTx co-staining (\%) }\end{array}$} \\
\cline { 2 - 3 } & WT & med \\
\hline $\begin{array}{l}\text { Multi-innervation } \\
\text { Preterminal axon branching points: } \\
\text { No branching point } \\
\begin{array}{l}\text { Mono branching point } \\
\text { Multi branching points }\end{array}\end{array}$ & 4 & $16^{*}$ \\
\hline
\end{tabular}

n.s., not significant; ${ }^{*} P<0.05$, Fischer test.

(Balice-Gordon \& Lichtman, 1993) is affected in the mutant. Next, we compared the branching pattern of pre-terminal axons, using confocal microscopy in association with deconvolution, in WT and mutant mice by counting the number of branching points formed by each axon contacting individual motor endplates. In the mutant, we observed a reduced branching of the distal axons visualized by NF staining (Table $1, P<0.05$, Fischer test; WT, $n=10$ and med, $n=47$ ). Distal axons failed to form the fine branching pattern characteristically found at normal NMJs (Fig 1B and E). In addition, preterminal axons in med mice appeared thick and swollen (Fig. 1E). However, our immunoblotting analysis showed no differences in NF and nAChR expression between WT or med mice at P19 (Fig. 1J). We further quantified the overlapping of nAChRs in terms of nerve terminal arborization. Motor endplates detected by $\alpha$-BgTx associated with SV2 (Fig. 2) and fasciculin2 (not shown) stainings were counted (Table 2 and Fig. 2). Analysis with the Fischer test showed no significant difference between the innervation of motor enplates in mutant and WT mice. This indicates that the clinical phenotype of med mice is not associated with denervation, Moreover, it is also most improbable that the terminal sprouting seen in the area free of endplates of terminally ill animals (Duchen, 1970; Musarella et al., 2006) is central to the med phenotype. Morphometric analysis was achevied by complementary quantification of partially innervated motor endplates using confocal microscopy in association with deconvolution. Quantification of partial overlap concerned only one of 208 NMJs in WT and five of 276 NMJs in mutants, underlining good overlapping of the postsynaptic compartment by the terminal arborization.

\section{NMJ organization was altered in med mice at P19}

Next, to focus on pre- and postsynaptic apparatus defects in the mutant, confocal microscopy in association with deconvolution (Fig. 1A, D and G) was strengthened by 3-D reconstruction analysis (Fig. 1B, C, E, F, H and I). In WT NMJs, the nAChR-rich postsynaptic apparatus is organized into a pretzel-shaped array of branches that precisely mirrors the finely branching pattern of the motor nerve terminal (Figs $1 \mathrm{~A}-\mathrm{C}$ and $2 \mathrm{~A}-\mathrm{D}$ ). Clusters of neurotransmitter-filled vesicles, visualized with SV2 staining, are organized into a wine grape-like shape, in precise juxtaposition to $\mathrm{nAChR}$ aggregates (Figs $1 \mathrm{~A}-\mathrm{C}$ and 2A-D). Abnormalities affecting the NMJs were identified in med mutants (Figs 1D-I and 2E-H). Presynaptic SV2 and postsynaptic nAChR stainings were more compact than in age-related WT mice and, in some cases, were not so precisely apposed (Figs 1E and $\mathrm{H}$ and $2 \mathrm{E}-\mathrm{H}$ ). Furthermore, poor terminal arborization was always detected in conjunction with defects in the postsynaptic apparatus defined by nAChR clusters lacking in structural complexity. Alto- 

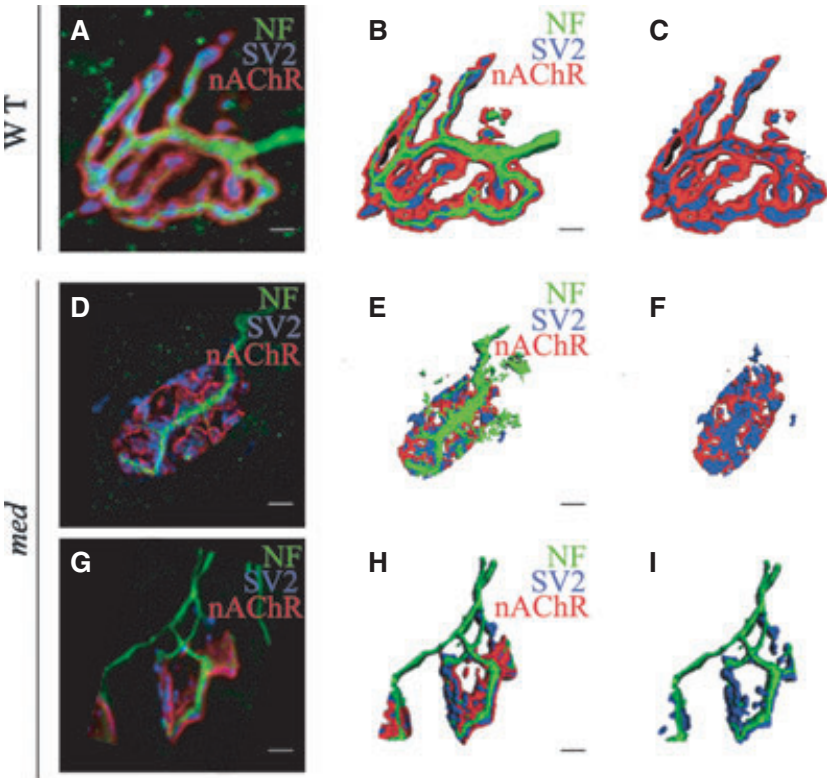

$\mathbf{F}$
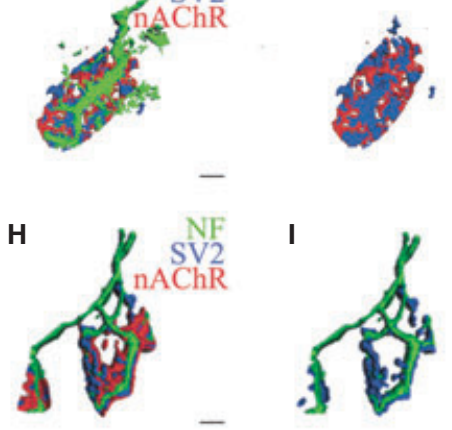

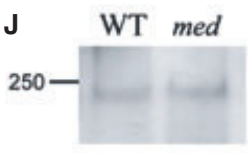

NF

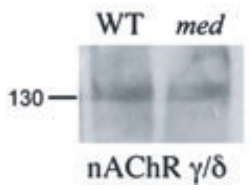

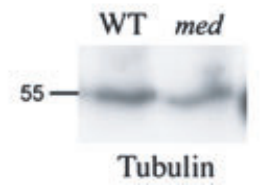

FIG. 1. NMJs are altered in terminally ill med mice (P19). (A-I) Co-staining of whole-mount ST muscles with NF and SV2 antibodies and labeled $\alpha$-BgTx; 14 WT and 34 med NMJs were analyzed by (A, D and G) deconvolution of confocal stacks and (B, C, E, F, H and I) 3-D visualization. (A-C) In WT muscles, motor nerve terminals $(\mathrm{NF}+\mathrm{SV} 2)$ fully occupied complex $\mathrm{nAChR}$ clusters $(\alpha-\mathrm{BgTx})$. The nAChR 'pretzel'-shaped is characteristic of mature endplates. Projection of 68 confocal planes $(11 \mu \mathrm{m})$. (D-F) In med muscles, many nAChR clusters have a 'spoon' shape, representing an immature morphology, absent in WT at this age. NMJs are smaller than those in controls and exhibit a poor branching of the axon terminals. Projection of 57 confocal planes $(9.12 \mu \mathrm{m})$. (G-I) Representative image of a med motor endplate (C-shaped staining by $\alpha$-BgTx; another type of immature morphology) innervated by two different axons. Projection of 152 confocal planes $(24.6 \mu \mathrm{m})$. (J) Immunoblotting analysis performed on total protein extracts of P19 WT and med SM muscles using NF, nAChR $\gamma / \delta$ subunits and $\beta$-tubulin antibodies Anti- $\beta$-tubulin antibody was used as a loading control. Scale bars, $2 \mu \mathrm{m}$.

gether, this analysis reveals that the organization of the innervated motor endplates, defined by the co-staining of pre- and postsynaptic components, was disorganized in mutant mice.

\section{NMJ maturation was delayed in med mice}

We then characterized how the NMJs were affected in med mice along development, at P7, P10 and P19, by morphometric analysis using confocal microscopy and 3-D reconstruction (Fig. 3). During this period of development, a complex transition from an ovoid plaque-like stucture to a branched, pretzel-like shape (Fig. 3A-C) occured whereas the area of the WT motor endplates remained constant (Fig. 3D). In contrast, in med mice at P19, we observed that the size of NMJs decreased: the area of NMJs were, on average, two-thirds the area of WT NMJs (195 vs. $289 \mu \mathrm{m}^{2}, P<0.001$, Student's $t$-test; Fig. 3D). In addition, the motor endplates were predominantly organized into either plaque-like or perforated (C-shaped) structures, corresponding to the morphology of younger motor endplates (at P19, $83 \%$ in mutant vs. $16 \%$ in WT, $P<0.001$, Fischer test; Fig. 3 A). At
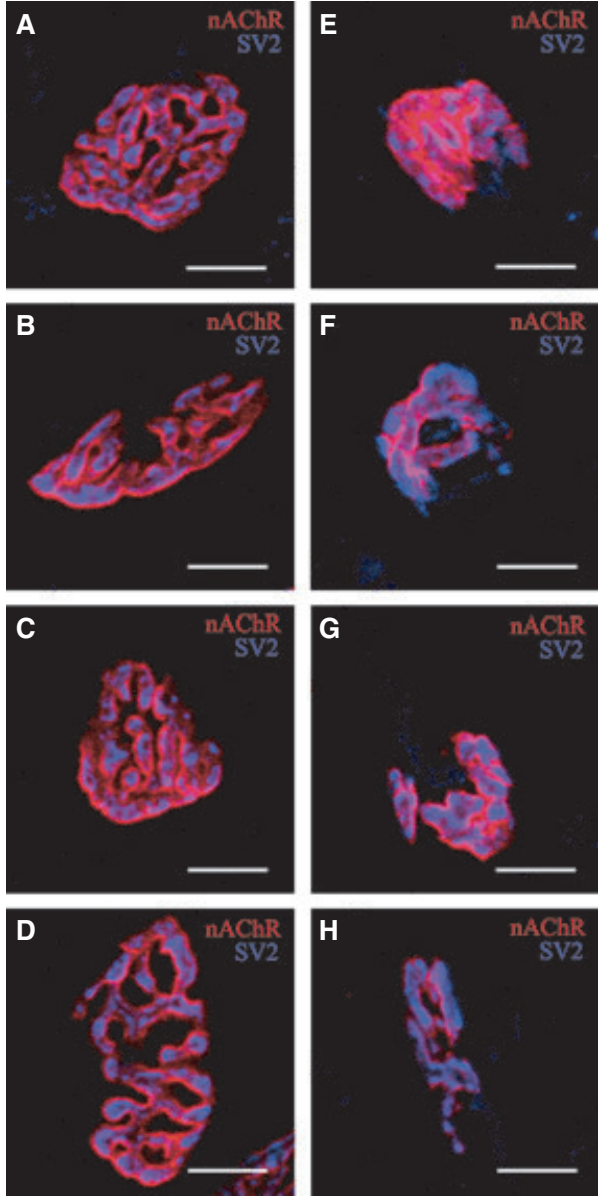

FIG. 2. Representative images of NMJ organization in P19 (A-D) WT and (E-H) med mice. Analysis by confocal microscopy and deconvolution of whole-mount ST muscles co-stained with SV2 antibodies and conjugated $\alpha$ BgTx. (A-D) In WT muscles, nerve terminal arborization overlie the postsynaptic nAChRs. Projection of 45 confocal planes $(17.6 \mu \mathrm{m})$. (E-H) In med muscles, the pre- and postsynaptic compartments are not precisely apposed but the overlap of nAChRs by terminal arborization is maintained. Projection of 71 confocal planes $(35 \mu \mathrm{m})$ except for the upper image (55 confocal planes, $27 \mu \mathrm{m})$. Scale bars, $8 \mu \mathrm{m}$.

P7, the percentage of each type of NMJ was similar in WT and in mutant mice (Fig. 3A). At P10, 28\% of the NMJs of WT animals displayed a branched shape corresponding to the morphology of a mature postsynaptic apparatus, whereas no such degree of maturation was observed in med mice $(P<0.001$, Fischer test; Fig. 3A). In addition, from $\mathrm{P} 7$ to $\mathrm{P} 10$, the percentage of ovoid plaques in WT decreased significantly whereas no change occured in the mutant (Fig. 3A). Altogether, these findings indicated that the synaptic

TABLE 2. Analysis of NMJ terminal arborization on ST muscles from P19 WT and med mice

\begin{tabular}{lll}
\hline & \multicolumn{2}{l}{$\begin{array}{l}\text { NMJs (\%) identified by } \\
\alpha \text {-BgTx staining }\end{array}$} \\
\cline { 2 - 3 } & WT & med \\
\hline SV2 co-staining & 73 & 78 (n.s.) \\
SV2 + Fasciculin2 co-staining & 73 & 68 (n.s.) \\
\hline
\end{tabular}

ns, not significant, Fischer test. WT: $\mathrm{n}=214$, med: $\mathrm{n}=336$ 
A
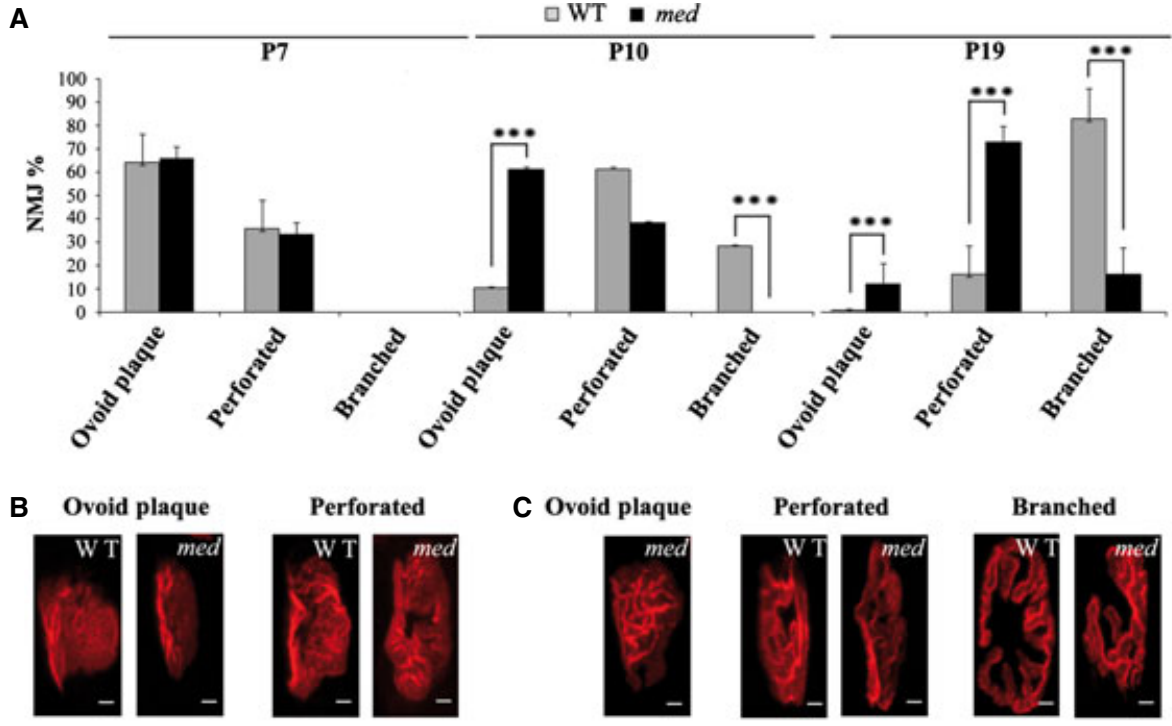

C Ovoid plaque
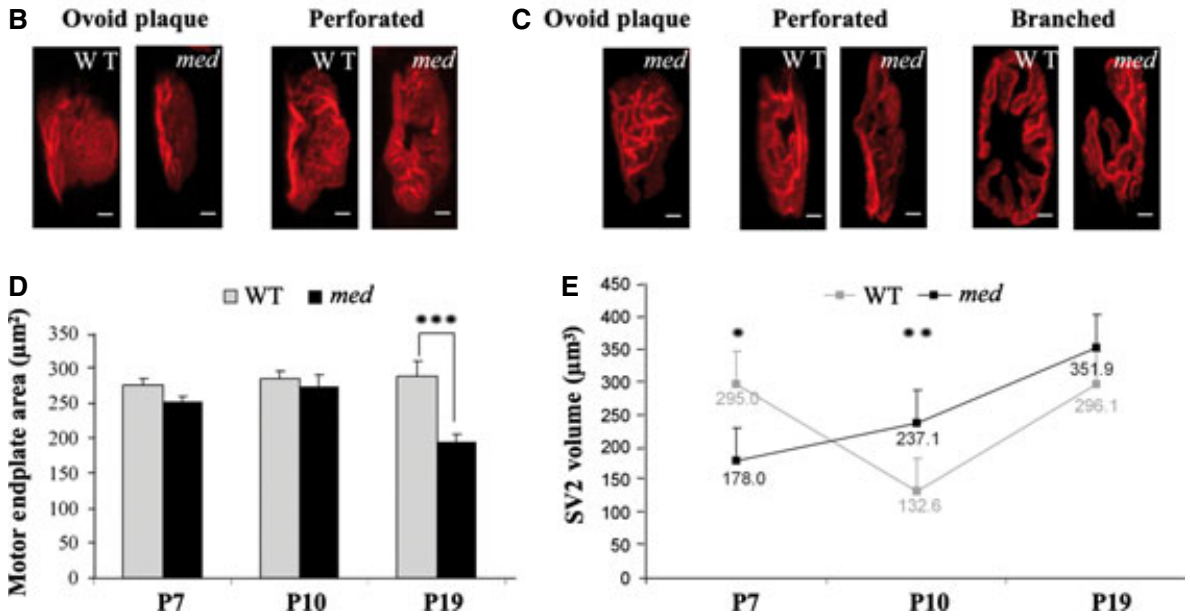

FIG. 3. The med mutation impairs pre- and postsynaptic specializations in developing NMJs. NMJs from at least two whole-mount ST muscles at each age were subjected to $\alpha$-BgTx and SV2 staining and analyzed by confocal microscopy and deconvolution, and in part by 3-D reconstruction. (A) Counts of immature (ovoid plaque type and sum of perforated and C-shaped types) and mature (branched type) motor endplates. Analysis of nAChR clusters induced by the med mutation, as compared to the WT, showed a persistent immature topology (***P<0.001, Fischer test). Results are shown as a percentage of the total number of WT and med NMJs analyzed at P7 (respectively, $n=81$ and 95), at P10 ( $n=36$ in both) and at P19 (respectively, $n=364$ and 428). (B) Representative images of the motor endplate organization at P7. Ovoid plaque type: confocal planes, $n=82(13.2 \mu \mathrm{m})$ and $n=73(11.7 \mu \mathrm{m})$ in WT and mutant respectively. Perforated type: confocal planes, $n=73(11.7 \mu \mathrm{m})$ and $n=42(6.7 \mu \mathrm{m})$ in WT and mutant respectively. (C) Representative images of the motor endplate organization at P19. Ovoid plaque type: confocal planes, $n=115(18.6 \mu \mathrm{m})$ in mutant. Perforated type: confocal planes, $n=43(6.84 \mu \mathrm{m})$ and $n=68(10.9 \mu \mathrm{m})$ in WT and mutant respectively. Branched type: confocal planes, $n=81(13 \mu \mathrm{m})$ and $n=38(6 \mu \mathrm{m})$ in WT and mutant respectively. (D) Motor endplate area quantification. No difference was observed between WT and med mice from P7 to P10. By contrast, in P19 med mice, NMJs were smaller than WT NMJs ( $* * * P<0.001$, Student's $t$-test). Results are shown as the mean \pm SD obtained from WT and med NMJs at P7 (respectively $n=68$ and 91), at P10 (respectively $n=77$ and 42 ), and at P19 (respectively $n=14$ and 34). (E) Quantification of the volume occupied by the SV2 staining. At P7 and P10, the presynaptic compartment staining showed significant difference between the WT and the mutant in the volume occupied by SV2-bearing vesicles (respectively $* P<0.05$ and $* * P<0.01$, Student's $t$-test). Results are shown as the mean \pm SD obtained from 10 NMJs in each condition. Scale bars in B and C, $2 \mu \mathrm{m}$.

maturation was delayed in med mice. To strengthen this conclusion, we detemined the volume of SV2 imunostaining, as an index of the volume occupied by the synaptic terminals, in developing NMJs (Fig. 3E). In WT, the developing SV2 volume demonstrated a biphasic curve, decreasing sharply from P7 to P10 then returning to its $\mathrm{P} 7$ value by P19. This is linked to remodelling of NMJs from multiple- to mono-innervation (Balice-Gordon \& Lichtman, 1993). In contrast, at P7, SV2 volume in the med mutant, which was significantly lower $(P<0.5$, Student's $t$-test $)$ than in the WT, increased gradually until P19 (Fig. 3E), suggesting an accumulation of synaptic vesicles, probably related to a failure of neuromuscular transmission (Mantilla et al., 2007).

\section{The number of TSCs decreased from the onset of the phenotype}

The number of TSCs at each NMJ increases rapidly during early postnatal life and then slows down in young adults (Hirata et al., 1997; Love \& Thompson, 1998). In contrast, we previously showed that the number of TSCs on ST muscle significantly decreased in terminally ill animals (Musarella et al., 2006). To determine how the TSC number was affected in med mice during development, we performed S100 immunochemistry combined with $\alpha$-BgTx staining at P7, P10 and P19, on two different muscles, the ST and the SM. Quantification of NMJs with associated TSCs (Fig. 4A; Tables 3 and 4) was performed on NMJs that could be observed in their totality. At P7, no difference was observed in all conditions; an average of $60 \%$ of NMJs were found to be associated with TSCs, a percentage already obtained by others (Hirata et al., 1997). At P10, the number of NMJs with TSCs was significantly different $(P<0.001$, Fischer test $)$ in the med mice in the two types of muscles, with a greater decrease in ST muscle (30\% of NMJs with TSCs in ST vs. 60\% in SM). This decrease persited to P19 $(P<0.001$, Fischer test $)$. Numbers of TSCs associated with each endplate (Fig. 4B; Tables 3 and 4) were counted by costaining S100 and DAPI, a nuclear marker, at each age on the two types of muscles. On average, NMJs had 2.5 associated TSCs in normal muscle, a number in accordance with that already reported (Hirata et al., 1997). Analysis with Student's $t$-test showed that the 
difference between TSC number per NMJ in mutant and WT (2.5) was highly significant $(P<0.001)$ at P10 (med SM, 1.7 and ST, 1.5) and increased until P19 (0.5 and 1.2). To examine the possibility that the decrease in TSC number was a result of cell death, we used the TUNEL technique to identify the nuclei of cells undergoing the DNA fragmentation characteristic of apoptosis. In ST muscle, TUNELDAPI co-labeled nuclei in S100-positive glial cells located in the vicinity of $\alpha$-BgTx-positive motor endplates were counted on thick
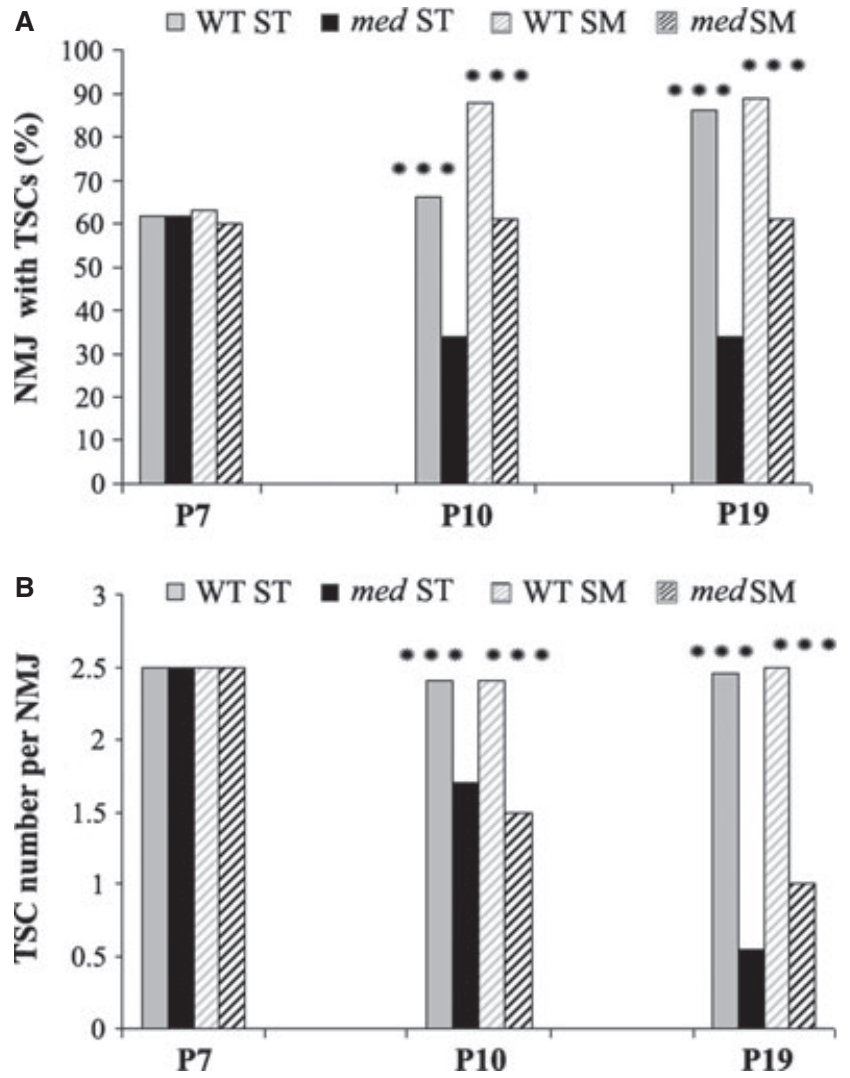

FIG. 4. The number of TSCs decreased from the onset of the clinical phenotype in the mutant. Co-labeling of ST and SM muscles from P7, P10 and P19 WT and med mice with an anti-S100 antibody, DAPI and labeled $\alpha$-BgTx. Counts of total number of NMJs, of NMJs with TSCs and of total number of TSCs are presented in Tables 3 and 4. (A) Percentage of NMJs with S100positive TSCs. (B) Mean number of S100-positive TSCs per NMJ. From P10 to P19, med mice displayed (A) a drastic decrease in number of NMJs with TSCs $(* * * P<0.001$, Fischer test) and (B) a reduced number of TSCs in TSCpositive junctions ( $* * * P<0.001$, Student's $t$-test).

TABLE 3. TSC number per NMJ on whole-mount of ST muscles from P7, P10 and P19 WT and med mice

\begin{tabular}{cccl}
\hline & NMJs & NMJs with TSC & TSCs \\
\hline P7 & & & \\
WT & 70 & 43 & 108 (n.s.) \\
med & 184 & 114 & 285 \\
P10 & & 50 & $120^{* * *}$ \\
WT & 75 & 44 & 75 \\
med & 149 & 186 & $465^{* * *}$ \\
P19 & 217 & 162 & 81 \\
WT & 486 & & \\
med & &
\end{tabular}

n.s., not significant; $* * * P<0.001$, Student's $t$-test. sections from P7, P10 and P19 WT and mutant mice (Fig. 5; Table 4). At P10, 2.5\% of remaining TSCs were TUNEL-positive (2722 cells, 68 apoptotic nuclei, seven muscles) in the WT, vs. $4.5 \%$ (1599 cells, 72 apoptotic nuclei, six muscles $)$ in the mutant $(P<0.001$, Chisquared test). As shown in Fig 4A and B, some motor endplates were already devoid of TSCs and others had less than their normal complement, suggesting that some TSCs had already completed apoptosis. Our data suggest that apoptosis might be responsible for the decrease in TSC number in med mice as observed after neonatal denervation where apoptotic TSCs are identifiably for only a short time and then disappear (Lubischer \& Thompson, 1999). In conclusion, med mice displayed a drastic decrease in TSCs (Fig. 4A), especially at TSC-positive junctions (Fig. 4B). Loss of TSCs occurs simultaneously with the establishment of the phenotype and is due, in part, to an increase in glial cell apoptosis.

\section{Discussion}

In this study we investigated the early cellular and molecular consequences of the med mutation on NMJs and charted the process of neuromuscular paralysis as the disease progressed. We observed that cellular defects preceded overt clinical phenotypes. The first detectable cellular effect of the mutation at the onset of the phenotype was a drastic reduction in the number of TSCs, in part due to an increase in glial apoptosis and delayed maturation of motor endplates. We also showed that in terminally ill animals mono-innervation was not achieved, synaptic vesicles accumulated in the presynaptic compartment and, finally, the size of motor endplates was reduced. All together, our findings suggested that the clinical weakness in these mutant mice could be due to structural abnormalities of the NMJ. Mammalian neuromuscular synapses undergo an activity-dependent competitive transition from multiple to single innervation during
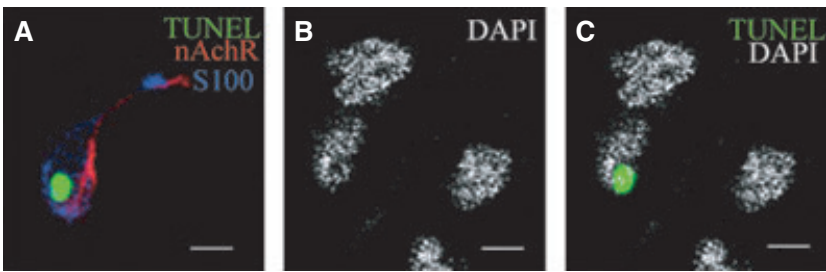

FIG. 5. Identification of apoptotic TSCs. TUNEL-DAPI co-labelled nucleus in an S100-positive TSC located in the vicinity of an $\alpha$-BgTx-positive motor endplate. Scale bars, $5 \mu \mathrm{m}$.

TABLE 4. Quantification of apoptotic TSCs on SM muscle thick sections from P7, P10 and P19 WT and med mice [Correction added after first online publication on 30 May 2012: The incorrect alignment of the table headers of Table 4 has been replaced.]

\begin{tabular}{cccccc}
\hline & NMJs & NMJs with TSC & TSCs & \multicolumn{2}{l}{ Apoptotic TSCs } \\
\cline { 5 - 6 } & & & & $n$ & $\%$ \\
\hline P7 & & & & & \\
WT & 640 & 403 & 1600 & 15 & 0.93 (n.s.) \\
med & 936 & 563 & 2340 & 21 & 0.89 \\
P10 & & & & & \\
WT & 1089 & 960 & 2722 & 68 & $2.50 * * *$ \\
med & 1066 & 646 & 1599 & 72 & 4.50 \\
P19 & & & & & \\
WT & 192 & 171 & 480 & 2 & 0.40 (n.s.) \\
med & 711 & 431 & 714 & 10 & 1.40 \\
\hline
\end{tabular}

n.s., not significant; $* * * P<0.001$, Chi-squared test. 
postnatal life (Thompson, 1983; Sanes \& Lichtman, 2001). Neural activity affects perinatal sorting and elimination of motor neuron inputs to skeletal muscle (Thompson, 1983; Sanes \& Lichtman, 2001; Personius \& Balice-Gordon, 2002; Personius et al., 2007). In fact, multiple innervation is maintained when activity is eliminated whereas enhanced activity accelerates the transition to single innervation (Thompson, 1983; Ridge \& Betz, 1984; Callaway et al., 1987; Ribchester, 1993). Here, we showed that in med mice the synaptic elimination was impeded, leading to a persistent multi-innervation. It is important to note that this persistent multi-innervation could lead to an underestimation of the impact of the mutation on the organization of the NMJ, even though the motor axon and motor endplate patterns are affected.

The maturation of the presynaptic element occurs between P7 and $\mathrm{P} 14$, a period during which there is a development of the axon terminal branches in phase with a segregation of synaptic vesicles in the active zones (Lupa \& Hall, 1989; Torres-Benito et al., 2011). In med mice, the maturation of the terminal arborization (SV2 staining) was delayed at P7, even before the onset of clinical signs (according to the criteria defined in the Materials and methods section). The volume occupied by SV2 was $40 \%$ lower than the SV2 volume measured in WT mice. However, after P7 we observed an increase in the size and density of releasable synaptic vesicle pools (the volume occupied by SV2 doubled between P7 and P19), probably related to a failure of neuromuscular transmission (Mantilla et al., 2007). By P19, med mice showed an imbalance in the coordinated growth of the presynaptic and postsynaptic elements, with an increase in synaptic vesicle pools and a reduction in the size of the motor endplates. Abnormalities of NMJ development have already been reported for Dok-7 and MuSK mice, which present reduced size of motor endplates and reduced postsynaptic folding (DeChiara et al., 1996; Beeson et al., 2006; Okada et al., 2006), but never for the med mutation. Moreover, P19 med mice also presented a significant decrease in the number of connections of the pre-terminal axon (NF staining) innervating each individual motor endplate, and a terminal axonal sprouting visible in regions devoid of endplates (Duchen, 1970; Musarella et al., 2006). Studies have shown that neuromuscular synaptic transmission is almost completely impaired in 17-day-old med mice (Duchen \& Stefani, 1971; Harris \& Pollard, 1986). This suggests that synaptic transmission deficits occur before P17. Therefore, med mouse phenotypes might be due to a deficit in neural activity, probably as a consequence of the association of both neurotransmission failures and NMJ structural alterations.

It is now clear that TSCs play a crucial role in NMJ development, regulating both pre- and postsynaptic maturations. This is demonstrated by the fact that in vivo selective ablation of TSCs in tadpole muscles leads to less synaptic growth, to more synaptic retractions and even to a complete loss of some synapses (Herrera et al., 2000; Reddy et al., 2003). Similarly, the lack of TSCs in ErB2-deficient mice or in neural crest cell-specific $\beta 1$-integrin-deficient mice lead to defective maturation in neuromuscular synaptogenesis (Lin et al., 2000; Pietri et al., 2004). In frog and mouse, Schwann cell-conditioned medium promotes postsynaptic maturation (Peng et al., 2003) via transforming growth factor- $\beta 1$ (Feng \& Ko, 2008b) and enhances spontaneous synaptic transmission in Xenopus nerve-muscle cultures and in situ in developing Xenopus NMJ (Cao \& Ko, 2007). TSCs also modulate the AChRs clustering at the motor endplate and the activation of nAChR genes in subsynaptic nuclei during development via the expression of Neuregulin-1 and -2, and agrin (Burgess et al., 1999; Yang et al., 2001; Rimer et al., 2004; Jaworski \& Burden, 2006). Finally, TSC proliferation and process growth, which is induced by axon-derived neuregulin-1, triggers nerve terminal sprouting in developing mouse muscles (Trachtenberg \& Thompson, 1997; Hayworth et al., 2006).
The number of TSCs at each NMJ increases rapidly during early postnatal life and precedes the expansion of the motor endplate size (Hirata et al., 1997; Love \& Thompson, 1998; Lubischer \& Bebinger, 1999). Any perturbations of TSC development and maturation in neonatal mice lead to the withdrawal of nerve terminals from synaptic sites (Trachtenberg \& Thompson, 1997). Previous studies showed that the number of TSCs at NMJs might be regulated by the musclederived neurotrophin-3, which possesses a developmentally regulated expression (Hess et al., 2007). Here, we observed that the first cellular effect of the med mutation was a decrease in the numbers of TSCs after the first week of birth. Therefore, it is likely that the reduction in TSCs in med mice during postnatal development may impair proper NMJ maturation. We also showed that reduction in TCS number might be due in part to an increase in glial apoptosis. However, the mechanisms modulating and/or triggering this reduction are still unknown. Thus, future studies should focus on deciphering the molecular mechanisms regulating the apoptosis of TSCs in med mice.

In conclusion, the med phenotype appears at a crucial stage of the NMJ development corresponding to the increase in TSC number, the elimination of multiple innervations and the synaptic maturation. In the med mice, these processes are altered such that there is a decrease in TSC number, persistent multiple innervations and a defect in synaptic maturation. All together these findings suggest that the med pathology is related to NMJ postnatal structural developmental defects, probably triggered by the progressive loss of the TSCs and an ineffective synaptic maturation.

\section{Supporting Information}

Additional supporting information can be found in the online version of this article:

Movie S1. Done at P10, showing how med mice are affected in their postnatal development at the onset of the phenotype. One med mouse and one WT mouse are shown.

Movie S2. Done at P19, showing how med mice are affected in their postnatal development at the terminal ill phenotype. Two med mice and one WT mouse are shown.

Please note: As a service to our authors and readers, this journal provides supporting information supplied by the authors. Such materials are peer-reviewed and may be re organized for online delivery, but are not copy-edited or typeset by Wiley-Blackwell. Technical support issues arising from supporting information (other than missing files) should be addressed to the authors.

\section{Acknowledgements}

This work was supported by grants from the AFM (Association Française contre les Myopathies, no. 11352). We thank M.-P. Blanchard (CMI, R.I.O. platform) for her help with the confocal microscopy, P. Marchot for conjugation and purification of fasciculin 2 and $\mathrm{C}$. Gomez for animal care.

\section{Abbreviations}

BSA, bovine serum albumin; med, motor endplate disease; nAChRs, nicotinic acetylcholine receptors; NF, neurofilament $\mathrm{H}$; NMJ, neuromuscular junction; $\mathrm{P}$, postnatal day; $\mathrm{PB}$, phosphate buffer; RT, room temperature; SM, sternomastoid; ST, sternal triangularis; SV2, synaptic vesicle protein 2; TSC, terminal Schwann cell; WT, wild type; $\alpha$-BgTx, $\alpha$-bungarotoxin.

\section{References}

Angaut-Petit, D., McArdle, J.J., Mallart, A., Bournaud, R., Pincon-Raymond, M. \& Rieger, F. (1982) Electrophysiological and morphological studies of a 
motor nerve in 'motor endplate disease' of the mouse. Proc. R. Soc. Lond. B Biol. Sci., 215, 117-125.

Araque, A., Parpura, V., Sanzgiri, R.P. \& Haydon, P.G. (1999) Tripartite synapses: glia, the unacknowledged partner. Trends Neurosci., 22, 208-215.

Auld, D.S. \& Robitaille, R. (2003) Glial cells and neurotransmission: an inclusive view of synaptic function. Neuron, 40, 389-400.

Balice-Gordon, R.J. \& Lichtman, J.W. (1993) In vivo observations of pre- and postsynaptic changes during the transition from multiple to single innervation at developing neuromuscular junctions. J. Neurosci., 13, 834-855.

Beeson, D., Higuchi, O., Palace, J., Cossins, J., Spearman, H., Maxwell, S., Newsom-Davis, J., Burke, G., Fawcett, P., Motomura, M., Muller, J.S., Lochmuller, H., Slater, C., Vincent, A. \& Yamanashi, Y. (2006) Dok-7 mutations underlie a neuromuscular junction synaptopathy. Science, 313, 1975-1978

Boiko, T., Rasband, M.N., Levinson, S.R., Caldwell, J.H., Mandel, G., Trimmer, J.S. \& Matthews, G. (2001) Compact myelin dictates the differential targeting of two sodium channel isoforms in the same axon. Neuron, 30, 91-104.

Brown, M.C., Holland, R.L. \& Ironton, R. (1980) Nodal and terminal sprouting from motor nerves in fast and slow muscles of the mouse. J. Physiol., 306 , 493-510.

Buckley, K. \& Kelly, R.B. (1985) Identification of a transmembrane glycoprotein specific for secretory vesicles of neural and endocrine cells. J. Cell Biol., 100, 1284-1294.

Burgess, D.L., Kohrman, D.C., Galt, J., Plummer, N.W., Jones, J.M., Spear, B. \& Meisler, M.H. (1995) Mutation of a new sodium channel gene, Scn8a, in the mouse mutant 'motor endplate disease'. Nat. Genet., 10, 461-465.

Burgess, R.W., Nguyen, Q.T., Son, Y.J., Lichtman, J.W. \& Sanes, J.R. (1999) Alternatively spliced isoforms of nerve- and muscle-derived agrin: their roles at the neuromuscular junction. Neuron, 23, 33-44.

Caldwell, J.H., Schaller, K.L., Lasher, R.S., Peles, E. \& Levinson, S.R. (2000) Sodium channel $\mathrm{Na}(\mathrm{v}) 1.6$ is localized at nodes of ranvier, dendrites, and synapses. Proc. Natl. Acad. Sci. USA, 97, 5616-5620.

Callaway, E.M., Soha, J.M. \& Van Essen, D.C. (1987) Competition favouring inactive over active motor neurons during synapse elimination. Nature, $\mathbf{3 2 8}$, 422-426.

Cao, G. \& Ko, C.P. (2007) Schwann cell-derived factors modulate synaptic activities at developing neuromuscular synapses. J. Neurosci., 27, 67126722

DeChiara, T.M., Bowen, D.C., Valenzuela, D.M., Simmons, M.V., Poueymirou, W.T., Thomas, S., Kinetz, E., Compton, D.L., Rojas, E., Park, J.S., Smith, C., DiStefano, P.S., Glass, D.J., Burden, S.J. \& Yancopoulos, G.D. (1996) The receptor tyrosine kinase MuSK is required for neuromuscular junction formation in vivo. Cell, $\mathbf{8 5}, 501-512$.

Dick, D.J., Boakes, R.J. \& Harris, J.B. (1985) A cerebellar abnormality in the mouse with motor end-plate disease. Neuropathol. Appl. Neurobiol., 11, 141-147.

Duchen, L.W. (1970) Hereditary motor end-plate disease in the mouse: light and electron microscopic studies. J. Neurol. Neurosurg. Psychiatry, 33, 23820

Duchen, L.W. \& Stefani, E. (1971) Electrophysiological studies of neuromuscular transmission in hereditary 'motor end-plate disease' of the mouse. $J$. Physiol., 212, 535-548.

Duchen, L.W. \& Strich, S.J. (1968) The effects of botulinum toxin on the pattern of innervation skeletal muscle in the mouse. Q. J. Exp. Physiol. Cogn. Med. Sci., 53, 84-89.

Duchen, L.W., Searle, A.G. \& Strich, S.J. (1967) An hereditary motor end-plate disease in the mouse. J. Physiol., 189, 4-6.

Feng, Z. \& Ko, C.P. (2008a) The role of glial cells in the formation and maintenance of the neuromuscular junction. Ann. N. Y. Acad. Sci., 1132, 1928.

Feng, Z. \& Ko, C.P. (2008b) Schwann cells promote synaptogenesis at the neuromuscular junction via transforming growth factor-beta1. J. Neurosci., 28, 9599-9609.

Feng, Z., Koirala, S. \& Ko, C.P. (2005) Synapse-glia interactions at the vertebrate neuromuscular junction. Neuroscientist, 11, 503-513.

Harris, J.B. \& Pollard, S.L. (1986) Neuromuscular transmission in the murine mutants "motor end-plate disease" and "jolting". J. Neurol. Sci., 76, 239253.

Hayworth, C.R., Moody, S.E., Chodosh, L.A., Krieg, P., Rimer, M. \& Thompson, W.J. (2006) Induction of neuregulin signaling in mouse schwann cells in vivo mimics responses to denervation. J. Neurosci., 26, 6873-6884.

Herrera, A.A., Qiang, H. \& Ko, C.P. (2000) The role of perisynaptic Schwann cells in development of neuromuscular junctions in the frog (Xenopus laevis). J. Neurobiol., 45, 237-254.
Hess, D.M., Scott, M.O., Potluri, S., Pitts, E.V., Cisterni, C. \& Balice-Gordon, R.J. (2007) Localization of TrkC to Schwann cells and effects of neurotrophin-3 signaling at neuromuscular synapses. J. Comp. Neurol., 501, 465-482.

Hirata, K., Zhou, C., Nakamura, K. \& Kawabuchi, M. (1997) Postnatal development of Schwann cells at neuromuscular junctions, with special reference to synapse elimination. J. Neurocytol., 26, 799-809.

Jaworski, A. \& Burden, S.J. (2006) Neuromuscular synapse formation in mice lacking motor neuron- and skeletal muscle-derived Neuregulin-1. $J$. Neurosci., 26, 655-661.

Kang, H., Tian, L. \& Thompson, W. (2003) Terminal Schwann cells guide the reinnervation of muscle after nerve injury. J. Neurocytol., 32, 975-985.

Kearney, J.A., Buchner, D.A., De Haan, G., Adamska, M., Levin, S.I., Furay, A.R., Albin, R.L., Jones, J.M., Montal, M., Stevens, M.J., Sprunger, L.K. \& Meisler, M.H. (2002) Molecular and pathological effects of a modifier gene on deficiency of the sodium channel Scn8a (Na(v)1.6). Hum. Mol. Genet., 11, 2765-2775.

Kohrman, D.C., Harris, J.B. \& Meisler, M.H. (1996) Mutation detection in the med and medJ alleles of the sodium channel Scn8a. Unusual splicing due to a minor class AT-AC intron. J. Biol. Chem., 271, 17576-17581.

Lin, W., Sanchez, H.B., Deerinck, T., Morris, J.K., Ellisman, M. \& Lee, K.F. (2000) Aberrant development of motor axons and neuromuscular synapses in erbB2-deficient mice. Proc. Natl. Acad. Sci. USA, 97, 1299-1304.

Love, F.M. \& Thompson, W.J. (1998) Schwann cells proliferate at rat neuromuscular junctions during development and regeneration. J. Neurosci., 18, 9376-9385.

Lubischer, J.L. \& Bebinger, D.M. (1999) Regulation of terminal Schwann cell number at the adult neuromuscular junction. J. Neurosci., 19, RC46.

Lubischer, J.L. \& Thompson, W.J. (1999) Neonatal partial denervation results in nodal but not terminal sprouting and a decrease in efficacy of remaining neuromuscular junctions in rat soleus muscle. J. Neurosci., 19, 8931-8944.

Lupa, M.T. \& Hall, Z.W. (1989) Progressive restriction of synaptic vesicle protein to the nerve terminal during development of the neuromuscular junction. J. Neurosci., 9, 3937-3945.

Mantilla, C.B., Rowley, K.L., Zhan, W.Z., Fahim, M.A. \& Sieck, G.C. (2007) Synaptic vesicle pools at diaphragm neuromuscular junctions vary with motoneuron soma, not axon terminal, inactivity. Neuroscience, 146, 178-189.

Musarella, M., Alcaraz, G., Caillol, G., Boudier, J.L., Couraud, F. \& AutilloTouati, A. (2006) Expression of Nav1.6 sodium channels by Schwann cells at neuromuscular junctions: role in the motor endplate disease phenotype. Glia, 53, 13-23.

Okada, K., Inoue, A., Okada, M., Murata, Y., Kakuta, S., Jigami, T., Kubo, S. Shiraishi, H., Eguchi, K., Motomura, M., Akiyama, T., Iwakura, Y., Higuchi, O. \& Yamanashi, Y. (2006) The muscle protein Dok-7 is essential for neuromuscular synaptogenesis. Science, 312, 1802-1805.

Peng, H.B., Yang, J.F., Dai, Z., Lee, C.W., Hung, H.W., Feng, Z.H. \& Ko, C.P (2003) Differential effects of neurotrophins and schwann cell-derived signals on neuronal survival/growth and synaptogenesis. J. Neurosci., 23, 50505060.

Personius, K.E. \& Balice-Gordon, R.J. (2002) Activity-dependent synaptic plasticity: insights from neuromuscular junctions. Neuroscientist, 8, 414422.

Personius, K.E., Chang, Q., Mentis, G.Z., O’Donovan, M.J. \& Balice-Gordon, R.J. (2007) Reduced gap junctional coupling leads to uncorrelated motor neuron firing and precocious neuromuscular synapse elimination. Proc. Natl. Acad. Sci. USA, 104, 11808-11813.

Pietri, T., Eder, O., Breau, M.A., Topilko, P., Blanche, M., Brakebusch, C., Fassler, R., Thiery, J.P. \& Dufour, S. (2004) Conditional beta1-integrin gene deletion in neural crest cells causes severe developmental alterations of the peripheral nervous system. Development, 131, 3871-3883.

Porter, J.D., Goldstein, L.A., Kasarskis, E.J., Brueckner, J.K. \& Spear, B.T. (1996) The neuronal voltage-gated sodium channel, $\operatorname{Scn} 8 \mathrm{a}$, is essential for postnatal maturation of spinal, but not oculomotor, motor units. Exp. Neurol., 139, 328-334.

Reddy, L.V., Koirala, S., Sugiura, Y., Herrera, A.A. \& Ko, C.P. (2003) Glial cells maintain synaptic structure and function and promote development of the neuromuscular junction in vivo. Neuron, 40, 563-580.

Ribchester, R.R. (1993) Co-existence and elimination of convergent motor nerve terminals in reinnervated and paralysed adult rat skeletal muscle. $J$. Physiol., 466, 421-441.

Ridge, R.M. \& Betz, W.J. (1984) The effect of selective, chronic stimulation on motor unit size in developing rat muscle. J. Neurosci., 4, 2614-2620.

Rimer, M., Prieto, A.L., Weber, J.L., Colasante, C., Ponomareva, O., Fromm, L., Schwab, M.H., Lai, C. \& Burden, S.J. (2004) Neuregulin-2 is synthesized by motor neurons and terminal Schwann cells and activates acetylcholine 
receptor transcription in muscle cells expressing ErbB4. Mol. Cell. Neurosci., 26, 271-281.

Rochon, D., Rousse, I. \& Robitaille, R. (2001) Synapse-glia interactions at the mammalian neuromuscular junction. J. Neurosci., 21, 3819-3829.

Sanes, J.R. \& Lichtman, J.W. (2001) Induction, assembly, maturation and maintenance of a post synaptic apparatus. Nat. Rev. Neurosci., 2, 791-805.

Son, Y.J., Trachtenberg, J.T. \& Thompson, W.J. (1996) Schwann cells induce and guide sprouting and reinnervation of neuromuscular junctions. Trends Neurosci., 19, 280-285.

Thompson, W.J. (1983) Lack of segmental selectivity in elimination of synapses from soleus muscle of new-born rats. J. Physiol., 335, 343-352.

Todd, K.J. \& Robitaille, R. (2006) Neuron-glia interactions at the neuromuscular synapse. Novartis Found. Symp., 276, 222-229; discussion 229-237, 275-281.

Todd, K.J., Darabid, H. \& Robitaille, R. (2010) Perisynaptic glia discriminate patterns of motor nerve activity and influence plasticity at the neuromuscular junction. J. Neurosci., 30, 11870-11882.

Torres-Benito, L., Neher, M.F., Cano, R., Ruiz, R. \& Tabares, L. (2011) SMN requirement for synaptic vesicle, active zone and microtubule postnatal organization in motor nerve terminals. PLOS ONE, 6, e26164.
Trachtenberg, J.T. \& Thompson, W.J. (1996) Schwann cell apoptosis at developing neuromuscular junctions is regulated by glial growth factor. Nature, 379, 174-177.

Trachtenberg, J.T. \& Thompson, W.J. (1997) Nerve terminal withdrawal from rat neuromuscular junctions induced by neuregulin and Schwann cells. $J$. Neurosci., 17, 6243-6255.

Vacher, H., Mohapatra, D.P., Misonou, H. \& Trimmer, J.S. (2007) Regulation of Kv1 channel trafficking by the mamba snake neurotoxin dendrotoxin $\mathrm{K}$. FASEB J., 21, 906-914.

Van Wart, A. \& Matthews, G. (2006) Expression of sodium channels Nav1.2 and Nav1.6 during postnatal development of the retina. Neurosci. Lett., 403, 315-317.

Vega, A.V., Henry, D.L. \& Matthews, G. (2008) Reduced expression of $\mathrm{Na}(\mathrm{v}) 1.6$ sodium channels and compensation by $\mathrm{Na}(\mathrm{v}) 1.2$ channels in mice heterozygous for a null mutation in Scn8a. Neurosci. Lett., 442, 6973.

Yang, J.F., Cao, G., Koirala, S., Reddy, L.V. \& Ko, C.P. (2001) Schwann cells express active agrin and enhance aggregation of acetylcholine receptors on muscle fibers. J. Neurosci., 21, 9572-9584. 